\title{
Selective thermal reduction of single-layer Mo03 nanostructures on Au(111)
}

\section{Citation}

Deng, Xingyi, Su Ying Quek, Monika M. Biener, Juergen Biener, Dae Hyuk Kang, Richard Schalek, Efthimios Kaxiras, and Cynthia M. Friend. 2008. "Selective Thermal Reduction of Single-Layer Mo03 Nanostructures on Au(111)." Surface Science 602 (6): 1166-74. https://doi.org/10.1016/ j.susc.2008.01.014.

\section{Permanent link}

http://nrs.harvard.edu/urn-3:HUL.InstRepos:41384018

\section{Terms of Use}

This article was downloaded from Harvard University's DASH repository, and is made available under the terms and conditions applicable to Open Access Policy Articles, as set forth at http:// nrs.harvard.edu/urn-3:HUL.InstRepos:dash.current.terms-of-use\#OAP

\section{Share Your Story}

The Harvard community has made this article openly available. Please share how this access benefits you. Submit a story.

Accessibility 
SUSC-D—06-00862, Submitted to Surf. Sci., Nov. 20, 2006

Revised, 10-May-07

\title{
Selective Thermal Reduction of Single-layer $\mathrm{MoO}_{3}$ nanostructures on $\mathrm{Au}(111)$
}

\author{
${ }^{1}$ Xingyi Deng, ${ }^{2} \mathrm{Su}$ Ying Quek, ${ }^{1,2,}$ Monika M. Biener, ${ }^{3,5}$ Juergen Biener, ${ }^{1}$ Dae Hyuk Kang, \\ ${ }^{3}$ Richard Schalek, ${ }^{2,4}$ Efthimios Kaxiras, ${ }^{1,2}$ Cynthia M. Friend* \\ ${ }^{1}$ Department of Chemistry and Chemical Biology, ${ }^{2}$ Division of Engineering and Applied \\ Sciences, ${ }^{3}$ Center for Nanoscale Science, and ${ }^{4}$ Department of Physics \\ Harvard University, Cambridge, MA 02138, USA \\ ${ }^{5}$ Nanoscale Synthesis and Characterization Laboratory, Lawrence Livermore National \\ Laboratory, Livermore, California 94550, USA
}

\begin{abstract}
$\mathrm{MoO}_{3}$ is an interesting oxide prototype because its catalytic activity is sensitive to the presence and nature of defects. In this work, we demonstrate that we can control the number of defects in single-layer $\mathrm{MoO}_{3}$ nanostructures grown on $\mathrm{Au}(111)$ by a simple thermal reduction treatment. X-ray photoelectron spectroscopy demonstrates the formation of $\mathrm{Mo}^{5+}$ species and oxygen vacancies during annealing at $650 \mathrm{~K}$. The percentage of $\mathrm{Mo}^{5+}$ increases with the duration of annealing, until a stable composition containing $50 \% \mathrm{Mo}^{6+}$ and $50 \% \mathrm{Mo}^{5+}$ is obtained. Surprisingly, the formation of lower oxidation states such as $\mathrm{Mo}^{4+}$ was not observed. The reduced $\mathrm{MoO}_{\mathrm{x}}$ islands remain one layer high, based on scanning tunneling microscope (STM) images. The two-dimensional nature of the reduced oxide nanocrystals may be due to a large barrier for structural reorganization and, thus, may account for the absence of Mo oxidation states lower than +5 . Based on scanning tunneling microscopy images and density functional calculations, we propose that the formation of $\mathrm{Mo}^{5+}$ ions during annealing is not associated with formation of oxygen point defects, but can be attributed to the formation of extended onedimensional shear defects. These reduced structures are useful for studying the dependence of reactivity on defect type, and present exciting possibilities for chemical sensors and other applications.
\end{abstract}

*Author to whom correspondence should be addressed. E-mail: cfriend@deas.harvard.edu.Keywords: X-ray photoelectron spectroscopy, Scanning tunneling Selective Thermal Reduction of Single-layer $\mathrm{MoO}_{3} \ldots$, Deng, etal. SUSC-D—06-00862 
microscopy, Defects, Molybdenum oxides, Density functional calculations, Nanostructures, $\mathrm{Au}(111)$, Catalysis

Selective Thermal Reduction of Single-layer $\mathrm{MoO}_{3} \ldots$, Deng, etal. SUSC-D—06-00862 


\section{Introduction}

Metal oxides constitute an important class of commercial catalysts [2]. For example, supported $\mathrm{MoO}_{3}$ promotes the partial oxidation of methane to formaldehyde and is widely used as a catalyst in the petroleum and chemical industry [3-6]. The catalytic properties of metal oxides depend sensitively on the presence and nature of defects. In the case of molybdenum oxides, a high surface oxidation state is expected to be a crucial factor for partial oxidation of hydrocarbons [7]; on the other hand, the presence of oxygen vacancies plays an important role in enhancing selectivity and reactivity by providing reactive sites [8]. Thus the ability to control quantity and type of defects present in the oxides is important, both for fundamental studies and for applications.

In recent years, novel metal oxide nanocrystals have been grown on metal surfaces[9] [Quek, $2005 \# 3805$;Biener, $2004 \# 3533]$. This development adds another dimension of interest because these nanocrystals often exhibit structures and electronic properties that are distinct from the bulk phase, and which arise from interface [Altieri, 2001 \#4007;Sinnott, $2003 \# 4008$ ] and nanoscale [10-12] effects. The ability to grow such structures in a controllable fashion thus opens the door for many novel applications. Previously, we demonstrated that crystalline twodimensional $\mathrm{MoO}_{3}$ nanostructures on $\mathrm{Au}(111)$ can be synthesized on $\mathrm{Au}(111)$ via oxidation of Mo nanoclusters using $\mathrm{NO}_{2}$ as oxidizing reagent [13], [14]. Whilst bulk $\mathrm{MoO}_{3}$ is a bilayered material, these $\mathrm{MoO}_{3}$ nanocrystals exhibit a unique single-layer structure with Au replacing the other half of the bilayer. Epitaxy with the $\mathrm{Au}(111)$ substrate is achieved via straining the in-plane Mo-O bonds which gives rise to electronic properties that are different from bulk $\mathrm{MoO}_{3}[1]$. The well-defined and novel structure of these nanocrystals provides an interesting starting point for the synthesis of reduced molybdenum oxide nanocrystals.

Selective Thermal Reduction of Single-layer $\mathrm{MoO}_{3} \ldots$, Deng, etal. SUSC-D—06-00862 
In this work, we demonstrate that it is possible to achieve selective reduction of these ordered $\mathrm{MoO}_{3}$ monolayer nanocrystals on $\mathrm{Au}(111)$.In principle, Mo has three stable oxidation states: $\mathrm{Mo}^{6+}, \mathrm{Mo}^{5+}$ and $\mathrm{Mo}^{4+}$. Recently, the group of Hrbek reported on the reduction of ramified $\mathrm{MoO}_{3}$ nanoclusters on the $\mathrm{Au}(111)$ surface prepared by $\mathrm{NO}_{2}$-induced oxidation of Mo nanoclusters at $500 \mathrm{~K}[15,16]$. The reduction of these $\mathrm{MoO}_{3}$ nanoclusters during annealing to $700 \mathrm{~K}$ was accompanied by a loss of Mo species, and resulted in a mixture of $\mathrm{Mo}^{+5}$ and $\mathrm{Mo}^{+6}$ species $[15,16]$. STM showed that the reduced $\mathrm{MoO}_{\mathrm{x}}$ structures remained 2-dimensional, but did not provide insight into the distribution of the reduced Mo atoms, in part because of structural disorder. Lower oxidation states of Mo such as $\mathrm{Mo}^{+4}$ can be prepared by oxidation of Mo metal by $\mathrm{O}_{2}$ exposure. In this case, the full oxidation to $\mathrm{Mo}^{+6}$ seems to be kinetically hindered.

Herein, we report on both theoretical and experimental studies on the thermal reduction of two-dimensional $\mathrm{MoO}_{3}$ nanocrystallites. Crystalline nanoparticles are more amenable to study using theory and experiment. As described in a previous publication, the synthesis of fully oxidized and well-ordered $\mathrm{MoO}_{3}$ nanostructures on $\mathrm{Au}(111)$ requires iterative Mo deposition and oxidation with $\mathrm{NO}_{2}$ [13]. Similar to bulk $\mathrm{MoO}_{3}$, the reduction of these single-layer $\mathrm{MoO}_{3}$ nanocrystals seems to proceed via shear defect formation which results from one-dimensional ordering and elimination of oxygen vacancies. The oxidation state of Mo associated with these shear-plane defects is +5 . The percentage of Mo in the +5 state increases as a function of annealing time, up to a maximum of $50 \%$ at $650 \mathrm{~K}$. This provides us with a methode to tailor the growth of reduced molybdenum oxide nanostructures with controllable oxidation states.

\section{Methods}

Selective Thermal Reduction of Single-layer $\mathrm{MoO}_{3} \ldots$, Deng, etal. SUSC-D—06-00862 


\section{Experimental}

The experiments were performed in three separate ultrahigh vacuum (UHV) systems, all described previously. Scanning tunneling microscopy experiments were performed in a commercial ultrahigh vacuum system with a base pressure of $5 \times 10^{-11}$ mbar. The system has separate compartments for sample preparation, e.g., Mo evaporation, and sample characterization. The characterization compartment is equipped with commercial instrumentation for scanning probe microscopy (Omicron), Auger electron spectroscopy (AES), and low energy electron diffraction (LEED).

The XPS chamber (base pressures $\sim 8 \times 10^{-10}$ mbar) is equipped with a low-energy electron diffraction (LEED) equipment, a quadrupole mass spectrometer (UTI 100C) for temperature programmed reaction experiments and an XPS system (PHI ESCA 5300) consisting of an X-ray source and a hemispherical analyzer.

The HREELS chamber (base pressure $\sim 5 \times 10^{-10} \mathrm{mbar}$ ) is equipped with a commercial highresolution electron energy loss spectrometer (LK technologies, model LK-2000-14-R), a LEED equipment, a quadrupole mass spectrometer (UTI 100C) and an Auger electron spectrometer (Perkin-Elmer model 15-555).

In all systems, the clean $\mathrm{Au}(111)$ surface was prepared by cycles of $\mathrm{Ar}^{+}$sputtering (1000 $\mathrm{eV}$ ) at $300 \mathrm{~K}$, followed by annealing at $900 \mathrm{~K}$ for 5 minutes and $700 \mathrm{~K}$ for 1 hour. This procedure was repeated until no impurities, e.g. carbon, oxygen, or Mo, were detected using either XPS or Auger electron spectroscopy. The presence of the 'herringbone' reconstruction, inherent to clean $\mathrm{Au}(111)$ surfaces, was verified by the presence of satellite spots in the $\mathrm{Au}(111)$ LEED patterns.

$\mathrm{MoO}_{3}$ nanostructures were prepared by oxidation of Mo nanoclusters using $\mathrm{NO}_{2}$. Deposition Selective Thermal Reduction of Single-layer $\mathrm{MoO}_{3} \ldots$, Deng, etal. SUSC-D_06-00862 
of Mo nanoclusers could be achieved either by chemical vapor deposition (CVD) or by physical vapor deposition (PVD). In the CVD method, $\mathrm{Mo}(\mathrm{CO})_{6}(98 \%)$ and $\mathrm{NO}_{2}$ (Matheson, 99.5\%) were dosed iteratively [13]: the reconstructed $\mathrm{Au}(111)$ surface was alternatively exposed to $1 \mathrm{~L}$ $\mathrm{Mo}(\mathrm{CO})_{6}$ and $10 \mathrm{~L} \mathrm{NO}_{2}$ at $450 \mathrm{~K}$. After four iterations, the sample was heated $(\mathrm{dT} / \mathrm{dt} \approx 5$ $\mathrm{K} /$ second) and maintained at $600 \mathrm{~K}$ for 1 minute. This cycle was repeated four times such that the total exposure to $\mathrm{Mo}(\mathrm{CO})_{6}$ and $\mathrm{NO}_{2}$ was $16 \mathrm{~L}$ and $160 \mathrm{~L}$, respectively. In the PVD method, molybdenum (Goodfellow, 99.9\%) was evaporated from rod material ( $\sim 1.5 \mathrm{~mm}$ diameter) using an electron beam evaporator (Omicron) $(900 \mathrm{~V} / \sim 65 \mathrm{~mA})$ followed by exposure to $\mathrm{NO}_{2}$ (Matheson, $99.5 \%$ ) at $500 \mathrm{~K}$ with a typical pressure of $1 \times 10^{-7}$ Torr, as described in detail elsewhere [14]. In the present studies, we used the CVD method to prepare $\mathrm{MoO}_{3}$ nanostructures in XPS and HREELS chambers and the PVD method in the STM system. Importantly, both methods yield identical $\mathrm{MoO}_{3}$ nanostructures, as confirmed by STM and LEED in earlier studies [13], [14].

All XPS spectra were collected at room temperature with a pass energy of $22.36 \mathrm{eV}$ using $\mathrm{Mg} \mathrm{K} \alpha$ radiation $\left((h v=1253.6 \mathrm{eV})\right.$. The binding energy was calibrated by $A u 4 \mathrm{f}_{7 / 2}=84.0 \mathrm{eV}$ for each spectrum. All HREELS (vibrational) spectra were collected at room temperature with a beam energy of $6.91 \mathrm{eV}$. The typical full width at half-maximum (FWHM) is $\sim 70 \mathrm{~cm}^{-1}$. STM images were collected at room temperature. $Z$-channel (topography) and $I$-channel (constant height) images were obtained simultaneously. Etched $\mathrm{Pt}_{0.8} \mathrm{Ir}_{0.2}$ tips from molecular imaging were used for imaging.

The XPS data analysis involved satellite and non-linear background subtraction, curvefitting (mix Gaussion-Lorentzian function with $85 \%$ of Gaussion) and peak area determination by integration of both Mo $3 \mathrm{~d}_{3 / 2}$ and $3 \mathrm{~d}_{5 / 2}$ peaks. The Mo $3 \mathrm{~d}$ region was fitted by doublets with Selective Thermal Reduction of Single-layer $\mathrm{MoO}_{3} \ldots$, Deng, etal. SUSC-D—06-00862 
fixed spectroscopic parameters, such as spin-orbit separation $(3.1 \mathrm{eV}), \operatorname{Mo}\left(3 \mathrm{~d}_{3 / 2}\right)-\left(3 \mathrm{~d}_{5 / 2}\right)$ intensity ratio $(\mathrm{R}=0.66)$ and full width at half maximum $(\mathrm{FWHM}=1.7 \mathrm{eV})$, but with independent and variable positions and intensities as optimized by the program.

\section{Theoretical}

Density functional theory (DFT) calculations employed the projected augmented wave method [Kresse, 1999 \#4010;Blochl, 1994 \#4011] and the Perdew-Wang 91 gradient correction for the exchange-correlation functional, as implemented in VASP [17]. We model the Au(111) surface by a slab of 6 Au layers, separated by $16.5 \AA$ of vacuum before the oxide is introduced, and the oxide and top $3 \mathrm{Au}$ layers were relaxed. Further computational details can be found in our previous publication [1].

\section{Results and discussion}

\section{As-prepared $\mathrm{MoO}_{3}$ nanostructures}

As described above, ordered two-dimensional $\mathrm{MoO}_{3}$ nanostructures on $\mathrm{Au}(111)$ can be produced via oxidation of Mo nanoclusters by $\mathrm{NO}_{2}$ exposure. The required Mo nanoclusters can be prepared by chemical vapor deposition (CVD) using molybdenum hexacarbonyl as a Mo precursor or by physical vapor deposition (PVD). Both techniques result in the growth of wellordered, 2 $\mathrm{D} \mathrm{MoO}_{3}$ islands as confirmed by STM and LEED [13][14]. For example, the characteristic $c(4 \times 2)$ LEED pattern displayed in Figure 1 was obtained from $\mathrm{MoO}_{3}$ nanostructures on $\mathrm{Au}(111)$ prepared by the CVD method in the XPS chamber. The pattern is identical to those observed in our earlier STM studies [13], indicating that the same structure was prepared.

Selective Thermal Reduction of Single-layer $\mathrm{MoO}_{3} \ldots$, Deng, etal. SUSC-D—06-00862 
XPS results demonstrate that the Mo in these oxide structures is essentially fully oxidized-in the +6 state-except for a small percentage of defects (Figure 2). Specifically, the predominant oxidation state is signified by the peaks with binding energies of $232.3 \mathrm{eV}$ (Mo $\left.3 \mathrm{~d}_{5 / 2}\right)$ and $235.4 \mathrm{eV}\left(\mathrm{Mo} \mathrm{3d}_{3 / 2}\right)$ which are similar to the binding energies reported for bulk $\mathrm{MoO}_{3}$ $\left(\mathrm{BE}=232.3-232.8 \mathrm{eV}\right.$; thus indicating the formation of $\mathrm{MoO}_{3}$. A weak shoulder at lower binding energy indicates the possible presence of a small percentage of $\mathrm{Mo}^{5+}$. Based on the signal intensities of the $\mathrm{Mo}^{+5}$ related peaks at 231.1 and $234.2 \mathrm{eV}$, as much as $6 \% \mathrm{Mo}^{5+}$ may be present.

The ability to nearly fully oxidize Mo so as to form ordered monolayer nanocrystals provides a starting point to control the oxidation states of these oxides. Iterative dosing of $\mathrm{Mo}(\mathrm{CO})_{6}$ and $\mathrm{NO}_{2}$ was necessary due to the kinetic control of oxidation: full oxidation of Mo is most readily effected with initially small Mo clusters $(<5 \mathrm{~nm})$ as starting material [13], whilst partially oxidized Mo with lower oxidation states, such as $\mathrm{Mo}^{5+}$ and $\mathrm{Mo}^{4+}$, was observed when Mo clusters were larger. Growth of larger $\mathrm{MoO}_{3}$ islands can be achieved by iterative deposition /oxidation cycles. For example, after 16 cycles well-ordered islands were found to be $600 \mathrm{~nm}^{2}$ on average, yielding a clear $c(4 \times 2)$ LEED pattern. No LEED pattern of $\mathrm{MoO}_{3}$ on $\mathrm{Au}(111)$ was reported by Z. Song et al.[16], possibly due to the ramified oxide structure, or smaller island size.

As mentioned above, a small fraction of $\mathrm{Mo}^{5+}$ was observed in the $\mathrm{MoO}_{3}$ nanostructures . Importantly, this small amount of $\mathrm{Mo}^{5+}$ persisted even after extensive dosing of $\mathrm{NO}_{2}$. In our study, $20 \mathrm{~L} \mathrm{NO}_{2}$ was used instead of $10 \mathrm{~L} \mathrm{NO}_{2}$ for each iterative dosing, and the resultant XP spectrum still yields about $6 \% \mathrm{Mo}^{5+}$. This result indicates the presence of a small percentage of defects in the structure, which has been observed in STM studies as well [14].

Selective Thermal Reduction of Single-layer $\mathrm{MoO}_{3} \ldots$, Deng, etal. SUSC-D—06-00862 
We have previously proposed a model for the atomic structure of a continuous 2D layer of single-layer $\mathrm{MoO}_{3}$ on $\mathrm{Au}(111)$. The model is consistent with LEED, STM (CVD and PVD), XPS (oxidation state: $\mathrm{Mo}^{6+}$ ) and HREELS results [1].

\section{Thermal reduction of $\mathrm{MoO}_{3}$ nanostructures at $650 \mathrm{~K}$}

The $\mathrm{Mo}^{5+}$ related XPS signal increases in intensity upon annealing to $650 \mathrm{~K}$ (Figure 3a). Specifically, annealing to $650 \mathrm{~K}$ for 20 minutes results in a broadening of Mo $3 \mathrm{~d}$ peaks, which can be fitted a mixture of $70 \% \mathrm{Mo}^{6+}$ and $30 \% \mathrm{Mo}^{5+}$ with binding energies of $232.5 \mathrm{eV}$ and 231.4 $\mathrm{eV}$ for Mo $3 \mathrm{~d}_{5 / 2}$, respectively. The fraction of Mo in the +5 oxidation state further increases with increasing annealing time, and ultimately a 1:1 mixture of $\mathrm{Mo}^{5+}: \mathrm{Mo}^{6+}$ is formed after annealing at $650 \mathrm{~K}$ for 1 hour (Figure 3a). No further changes in the $\mathrm{Mo}^{5+}: \mathrm{Mo}^{6+}$ ratio were observed upon heating for longer times, up to 120 minutes (Figure 3b). Furthermore, no other oxidation states were detected after heating to $650 \mathrm{~K}$ in any of our experiments. Specifically, no $\mathrm{Mo}^{4+}$ is formed, based on the absence of intensity in the Mo $3 \mathrm{~d}_{5 / 2}$ region at $229.1 \mathrm{eV}$.

The partial reduction of $\mathrm{Mo}^{+6}$ to $\mathrm{Mo}^{+5}$ is accompanied by the loss of a small amount of Mo based on the integrated intensities of Mo 3d region (Figure 3b). Specifically, the total amount of Mo decrease by $\sim 6 \% / 8 \% / 10 \%$ during annealing at $650 \mathrm{~K}$ for $20 / 40 / 60$ minutes. Finally, $88 \%$ Mo remains on the Au surface upon annealing at $650 \mathrm{~K}$ for 120 minutes. The small loss of Mo is probably the result of some dissolution of Mo into the bulk associated with reduction or desorption of $\mathrm{MoO}_{3}$ molecules during annealing.

The partial reduction of $\mathrm{Mo}^{+6}$ during annealing also causes changes in the vibrational spectra (Figure 4). Before annealing, the most intense peak is found at $850 \mathrm{~cm}^{-1}$ with a shoulder at $990 \mathrm{~cm}^{-1}$ (Figure 4(i)), and two less intense peaks are observed at 480 and $280 \mathrm{~cm}^{-1}$. Based on Selective Thermal Reduction of Single-layer $\mathrm{MoO}_{3} \ldots$, Deng, etal. SUSC-D—06-00862 
DFT calculations on the $\mathrm{MoO}_{3}$ structure, the peaks at $990 \mathrm{~cm}^{-1}$ and $850 \mathrm{~cm}^{-1}$ can be assigned to stretches of the terminal $\mathrm{O}$ and bridging $\mathrm{O}$ bonds respectively, whilst the other modes involve the bending of bridging $\mathrm{O}$ bonds, and to a lesser extent, terminal $\mathrm{O}$ bonds. The atomic structure of the ordered $\mathrm{MoO}_{3}$ monolayer phase on $\mathrm{Au}(111)$ including the positions of terminal and bridging oxygen atoms is shown in Figure 5(a). Annealing the $\mathrm{MoO}_{3}$ nanostructures to $650 \mathrm{~K}$ results in a decrease of the peak intensities (Figure 4(ii)-(iv)). Specifically, the peak intensity of the $850 \mathrm{~cm}^{-1}$ bridging $\mathrm{O}$ stretch deceases to about $80 \%$ after 20 minutes of annealing, and to about $20 \%$ after 60 minutes. The lower frequency peaks also decrease. Meanwhile, the terminal oxygen stretch peak resolves upon annealing. Although a decrease in peak intensity is also observed for the terminal $\mathrm{O}$ stretch, the ratio of peak intensities for the bridging and terminal $\mathrm{O}$ stretches changes from $4: 1$ to $2: 1$, indicating a structural change. The fact that the terminal oxygen related vibrational modes are less affected by the reduction process is a first indication that the reduced Mo oxide remains a two-dimensional structure

\section{Proposed model for thermally reduced oxide}

We propose that reduction of the $\mathrm{MoO}_{3}$ monolayer structures involves the formation of shear defects similar to of the shear planes found in the so-called Magneli phases of reduced bulk Mo-trioxide, $\mathrm{Mo}_{n} \mathrm{O}_{3 n-1}$ [18]. Such shear defects could eliminate one-dimensionally ordered arrays of oxygen vacancies formed during annealing by a local transformation (shearing) from cornerconnected to edge-connected $\mathrm{MoO}_{3}$ units. [lit20] In the case of the Magneli phases of reduced bulk Mo-trioxide, the shear planes are regularly spaced indicating a mutual elastic repulsion.. The driving force for shear plane formation seems to be the stabilization of isolated oxygen

Selective Thermal Reduction of Single-layer $\mathrm{MoO}_{3} \ldots$, Deng, etal. SUSC-D—06-00862 
vacancies by migration towards one another to form shear planes of edge-sharing $\mathrm{MoO}_{6}$ octahedra. The shear planes can then expand by trapping more vacancies [18].

Similarly, the reduction of $2 \mathrm{D} \mathrm{MoO}_{3}$ islands could cause the formation of one-dimensional shear defects which would lead to the elimination of the oxygen vacancies formed during thermal reduction. The formation of these shear defects involves a local transformation of corner-connected $\mathrm{MoO}_{4}$ tetrahedra to edge-connected $\mathrm{MoO}_{4}$ tetrahedra (Figures $5 \mathrm{~b}$ and 6e). This scenario seems to be supported by STM. Figure 6 shows STM images collected from $\mathrm{MoO}_{3^{-}}$ covered $\mathrm{Au}(111)$ surfaces before (a) and after annealing at $\mathrm{T}>650 \mathrm{~K}(\mathrm{~b}-\mathrm{d})$. The stoichiometric $\mathrm{MoO}_{3}$ islands shown in Figure 6a were prepared by PVD of Mo ( $\left.\sim 0.2 \mathrm{ML}\right)$ and subsequent oxidation with $\mathrm{NO}_{2}$ at $500 \mathrm{~K}$. These $\mathrm{MoO}_{3}$ islands exhibit an apparent height of $\sim 0.5 \mathrm{~nm}$ [14]. Annealing at $\mathrm{T}>650 \mathrm{~K}$ leads to several changes: (i) the $\mathrm{MoO}_{3}$ islands grow considerably in size (Figure 6b); (ii) extended, one-dimensional defects appear on top of the $\mathrm{MoO}_{3-\mathrm{x}}$ islands (Figure 6c) which (iii) separate regions shifted by half a lattice constant (Figure 6d), and (iv) the Mo coverage decreases by $\sim 60 \%$ (suggesting that the actual annealing temperature was slightly higher than $650 \mathrm{~K}$ ). Clearly, the reduced Mo oxide has a two-dimensional structure despite the large scale mass transport required for the observed morphological changes. The Z-channel STM image shown in Figure 6c reveals that the surface corrugation on top of the reduced $\mathrm{MoO}_{3}$ island is less than $0.1 \mathrm{~nm}$. A possible structural model of a reduced single-layer $\mathrm{MoO}_{3}$ island is shown in Figure 6e. The model assumes the formation of shear planes by a local transformation of corner connected $\mathrm{MoO}_{3}$ units to edge connected $\mathrm{MoO}_{3}$ units, and is capable of reproducing the main features observed in the high resolution STM image shown in Figure 6d. Specifically, it predicts the observed shift of the $\mathrm{MoO}_{3}$ lattice by half a lattice constant between regions separated by a shear defect. The average spacing between the line defects shown in Figure $6 \mathrm{c}, \mathrm{d}$ is Selective Thermal Reduction of Single-layer $\mathrm{MoO}_{3} \ldots$, Deng, etal. SUSC-D—06-00862 
approximately $1.5 \mathrm{~nm}$ which according to the shear model shown in Figure 6e would correspond to $\mathrm{Mo}^{+5}$ fraction of $40 \%$.

Figure 7 shows a relaxed shear defect structure in which one bridging $\mathrm{O}$ is lost for every four Mo. The defect is characterized by more closely-spaced Mo rows connected by 3-fold coordinated $\mathrm{O}$ (3-fold coordinated $\mathrm{O}$ is also present in bulk $\mathrm{MoO}_{3}$ ). The bond lengths of Mo-O and $\mathrm{Mo}-\mathrm{O}_{\mathrm{b}}$ in the defect structure are, respectively, in the range $(-5.1,+5.1) \%$ and $(-4.6,+6.2) \%$ of their corresponding values in the fully oxidized oxide. The bond length of Mo- $\mathrm{O}_{\mathrm{t}}$ remains unchanged consistent with the vibrational spectra shown in Figure 4. The longest Mo-O bonds $(2.08 \AA)$ are those joining $\mathrm{Mo}^{5+}$ to a 3 -fold coordinated $\mathrm{O}$ atom across the defect line. The formal oxidation state of Mo is +6 and +5 between and along the shear defects respectively, and no $\mathrm{Mo}^{4+}$ is present. This structure is consistent with X-ray photoelectron data. The loss of bridging $\mathrm{O}$ in the shear structure is also consistent with vibrational peak intensities, which indicate a sharper drop in the intensity for the bridging $\mathrm{O}$ stretch compared to that for the terminal $\mathrm{O}$ stretch. The formation of shear defects is further corroborated by the observation that the lattice planes on both sides of these defects are shifted by half a lattice constant with respect to each other (Figure 6). Finally, the 1D defects imaged in STM (Figure 6) are in approximately the same orientation as predicted by our model; that is in the experimental STM image, these defects are at an angle of about $42^{\circ}$ relative to the $<1-10>$ direction of Au. Simulating STM images in the Tersoff-Hamann approximation results in a bright line representing the shear plane defect, which makes an angle of $41^{\circ}$ relative to the $<1-10>$ direction, in agreement with experiment (Figure $7 \mathrm{c}$ ).

From the projected density of states of the theoretical model (Figure 8), it can be seen that Mo atoms associated with the shear defect have a higher density of states at the Fermi level than those away from the defect or those in the fully-oxidized oxide, suggesting possible enhanced Selective Thermal Reduction of Single-layer $\mathrm{MoO}_{3} \ldots$, Deng, etal. SUSC-D—06-00862 
reactivity (especially involving loss of $\mathrm{O}$ ) along shear planes. The predominant contribution to Fermi level states arises from d states of $\mathrm{Mo}^{5+}$ (Figure 8a). The density of states of $\mathrm{O}$ bridging atoms in the shear plane region, both of $\mathrm{O}_{\mathrm{a}}$ and $\mathrm{O}_{\mathrm{b}}$ type, are significantly shifted compared to those of corresponding sites far from the defect. This is the result of an increase in coordination number of these $\mathrm{O}$ atoms ( 2 away from the defect and 3 in the defect region), and changes in the oxidation state of Mo atoms in the defect region, to which these $\mathrm{O}$ bridging atoms are bonded.

The shear defects may exhibit different reactivity and interesting electrical properties due to the presence of the oxygen vacancies. The ability to quantitatively control defects and oxidation states is the key toward demonstrating the effect of defects and specific oxidation states on oxidation reactions. Our results above clearly show that single-layer $\mathrm{MoO}_{3}$ nanostructures on $\mathrm{Au}(111)$ can be selectively reduced in a controllable manner that depends on annealing temperature and duration. In addition, the resulting molybdenum oxide structures may also present exciting possibilities for other applications, e.g. chemical sensors.

\section{Limitations of model and other possible structures}

It is important to note that the theoretical model proposed here may not accurately represent the experimental system, because the model assumes an infinitely long defect structure. This difference may account for discrepancies between the STM simulation and experimental image, such as the shorter length of defects and presence of black spots in the experimental image, which also contains a smaller density of defects. Systems involving finite-length-defects or lower densities of defects are more complex and computationally demanding. However, the model represents a possible local atomic structure at the defects and is consistent with experimental results.

Selective Thermal Reduction of Single-layer $\mathrm{MoO}_{3} \ldots$, Deng, etal. SUSC-D_06-00862 
The final composition of 1:1 for the ratio of $\mathrm{Mo}^{5+}$ to $\mathrm{Mo}^{6+}$, obtained with prolonged annealing, corresponds to the well-defined stoichiometry $\mathrm{Mo}_{4} \mathrm{O}_{11}$. It is interesting that bulk $\mathrm{Mo}_{4} \mathrm{O}_{11}$ is the Magneli phase with the highest known density of shear defects [18]. Bulk $\mathrm{Mo}_{4} \mathrm{O}_{11}$ has a three-dimensional (3D) structure, with layers of $\mathrm{Mo}_{6} \mathrm{O}_{22}$ linked by $\mathrm{MoO}_{4}$ tetrahedra via covalent bonds [Canadell, $1989 \# 4012$ ]. It is possible that the final stable $\mathrm{Mo}_{4} \mathrm{O}_{11}$ phase observed in our experiments is the two-dimensional analog to the three-dimensional $\mathrm{Mo}_{4} \mathrm{O}_{11}$ bulk phase. This would present exciting opportunities for fundamental studies because bulk $\mathrm{Mo}_{4} \mathrm{O}_{11}$ exhibits charge density wave transitions [Canadell, $1989 \# 4012$ ], and it would be interesting to determine how a metallic substrate would affect this phenomenon. The fact that we do not observe the formation of a three-dimensional $\mathrm{Mo}_{4} \mathrm{O}_{11}$ phase indicates the presence of kinetic barriers. Indeed, a 3D Mo oxide phase can be grown on $\mathrm{Au}(111)$ if the Mo deposition is performed in an ambient of $\mathrm{NO}_{2}$ at $500 \mathrm{~K}$ (unpublished results)..Further STM studies will be necessary to refine the structural model described in this work.

\section{Conclusions}

Well-ordered Mo oxide nanostructures on $\mathrm{Au}(111)$ have been prepared via deposition of metallic Mo (CVD or PVD) followed by oxidation using $\mathrm{NO}_{2}$ as a reactant. The observation of a well-ordered $c(4 \times 2)$ LEED pattern proves the formation of a crystalline epitaxial Mo oxide. XPS indicates that this oxide is $\mathrm{MoO}_{3}$, with up to $6 \%$ of $\mathrm{Mo}^{5+}$ defects. Upon annealing at $650 \mathrm{~K}$, these $\mathrm{MoO}_{3}$ nanostructures are selectively reduced to $\mathrm{Mo}^{5+}$ indicating the formation of oxygen vacancies. The percentage of $\mathrm{Mo}^{5+}$ and the corresponding oxygen vacancies increases with increasing annealing time until the stable composition $\mathrm{Mo}_{4} \mathrm{O}_{11}\left(50 \% \mathrm{Mo}^{+5}\right)$ is reached after one hour at $650 \mathrm{~K}$. Further reduction towards $\mathrm{Mo}^{4+}$ has not been observed. This simple annealing

Selective Thermal Reduction of Single-layer $\mathrm{MoO}_{3} \ldots$, Deng, etal. SUSC-D—06-00862 
treatment allows one to fine-tune the concentration of $\mathrm{Mo}^{5+}$ in the resulting selectively reduced structures by controlling the temperature and annealing time.

Based on our preliminary STM results, the thermal reduction of $\mathrm{MoO}_{3}$ nanostructures on $\mathrm{Au}$ seems to lead to the formation of shear defects, similar to those observed in reduced bulk $\mathrm{MoO}_{3}$. The driving force for the formation of these shear defects could be the elimination of oxygen vacancies produced by annealing. However, in contrast to the 3D structures observed in reduced bulk $\mathrm{MoO}_{3}$, the reduced $\mathrm{MoO}_{3}$ islands on $\mathrm{Au}(111)$ remain pure 2D structures..The formation of 3D structures in our model system has only been observed if Mo deposition is performed in an ambient of $\mathrm{NO}_{2}$ Thus the reduced 2D Mo oxide structures described in this paper are distinctly different from the 3D structures found in reduced bulk $\mathrm{MoO}_{3}$, and therefore provide an interesting platform for further studies. The ability to control and tailor the oxidation states in these novel oxide structures on metal surfaces suggests exciting possibilities for future applications. Further STM studies of the final $\mathrm{Mo}_{4} \mathrm{O}_{11}$ structure will be necessary to refine the structural model.

\section{Acknowledgements}

This work was supported by the National Science Foundation, under the Harvard Nanoscale Science and Engineering Center, PHY-011-7795, and the Department of Energy, Basic Energy Sciences, under FG02-84-ER13289. Part of the work by J.B. and M.M.B. was performed under the auspices of the U. S. Department of Energy by the University of California, Lawrence Livermore National Laboratory under Contract W-7405-Eng-48. S.Y.Q. thanks the Singapore

Selective Thermal Reduction of Single-layer $\mathrm{MoO}_{3} \ldots$, Deng, etal. SUSC-D—06-00862 
Agency for Science, Technology and Research for a graduate fellowship. The calculations were performed on the National Computational Science Alliance IBM P690, under DMR030044.

Selective Thermal Reduction of Single-layer $\mathrm{MoO}_{3} \ldots$, Deng, etal. SUSC-D—06-00862 


\section{Figure Captions}

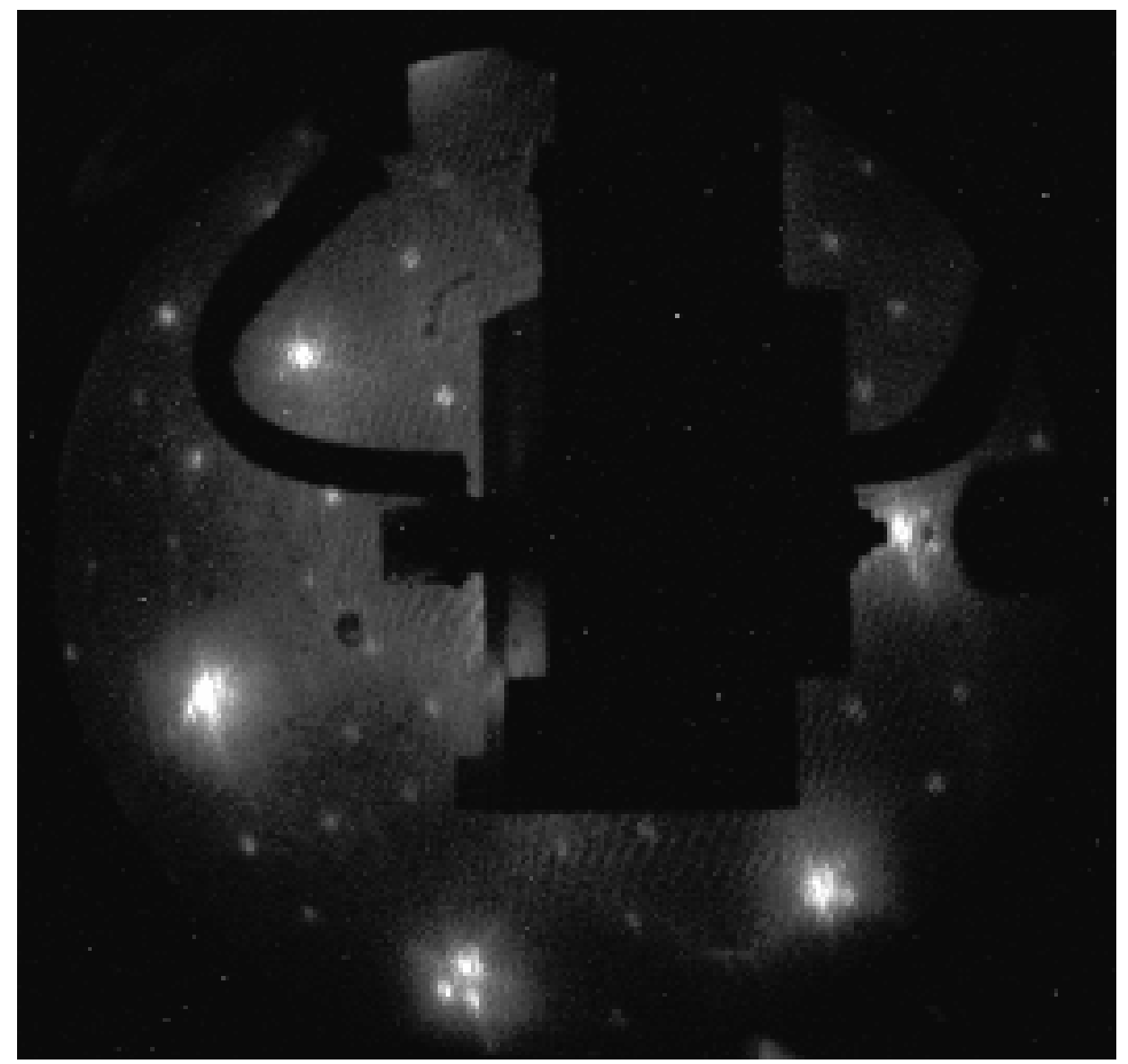

Figure 1. LEED patterns obtained at $300 \mathrm{~K}$ showing a $\mathrm{c}(4 \times 2)$ structure after iterative dosing. The beam energy was $77 \mathrm{eV}$. This pattern is identical to that previously reported [13], [14].

Selective Thermal Reduction of Single-layer $\mathrm{MoO}_{3} \ldots$, Deng, etal. SUSC-D—06-00862 


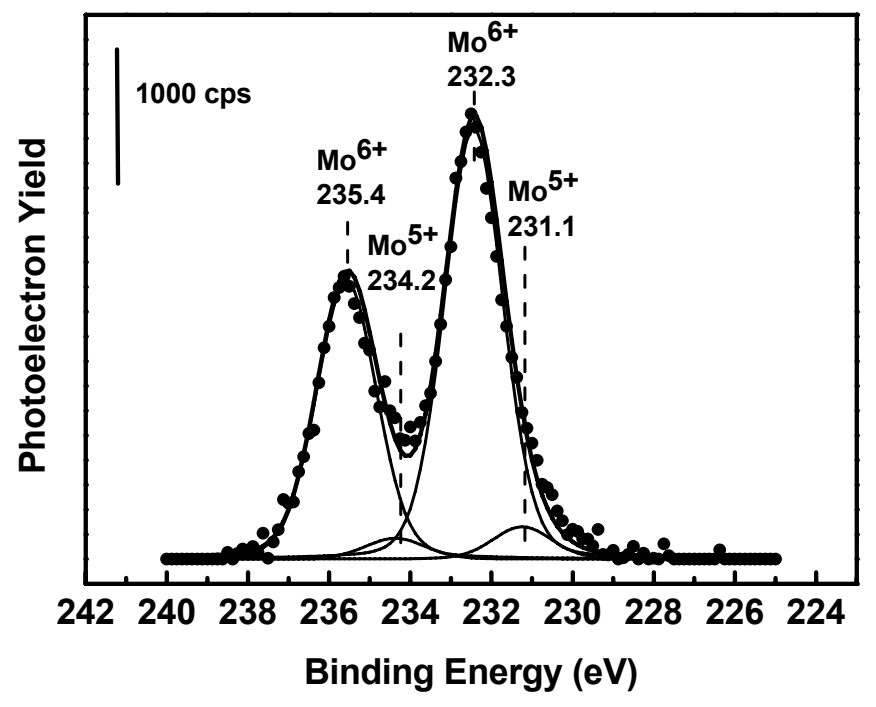

Figure 2. X-ray photoelectron spectrum for the Mo 3d region obtained after preparation of the oxidized Mo nanocrystals on $\mathrm{Au}(111)$ using iterative dosing of $\mathrm{Mo}(\mathrm{CO})_{6}$ and $\mathrm{NO}_{2}$. The total spectrum was fitted by $\sim 94 \% \mathrm{Mo}^{6+}\left(3 \mathrm{~d}_{5 / 2}, 232.3 \mathrm{eV}\right)$ and $\sim 6 \% \mathrm{Mo}^{5+}\left(3 \mathrm{~d}_{5 / 2}, 231.1 \mathrm{eV}\right)$. The curve fitting parameters and methods were described in detail in the experimental section.

Selective Thermal Reduction of Single-layer $\mathrm{MoO}_{3} \ldots$, Deng, etal. SUSC-D—06-00862 

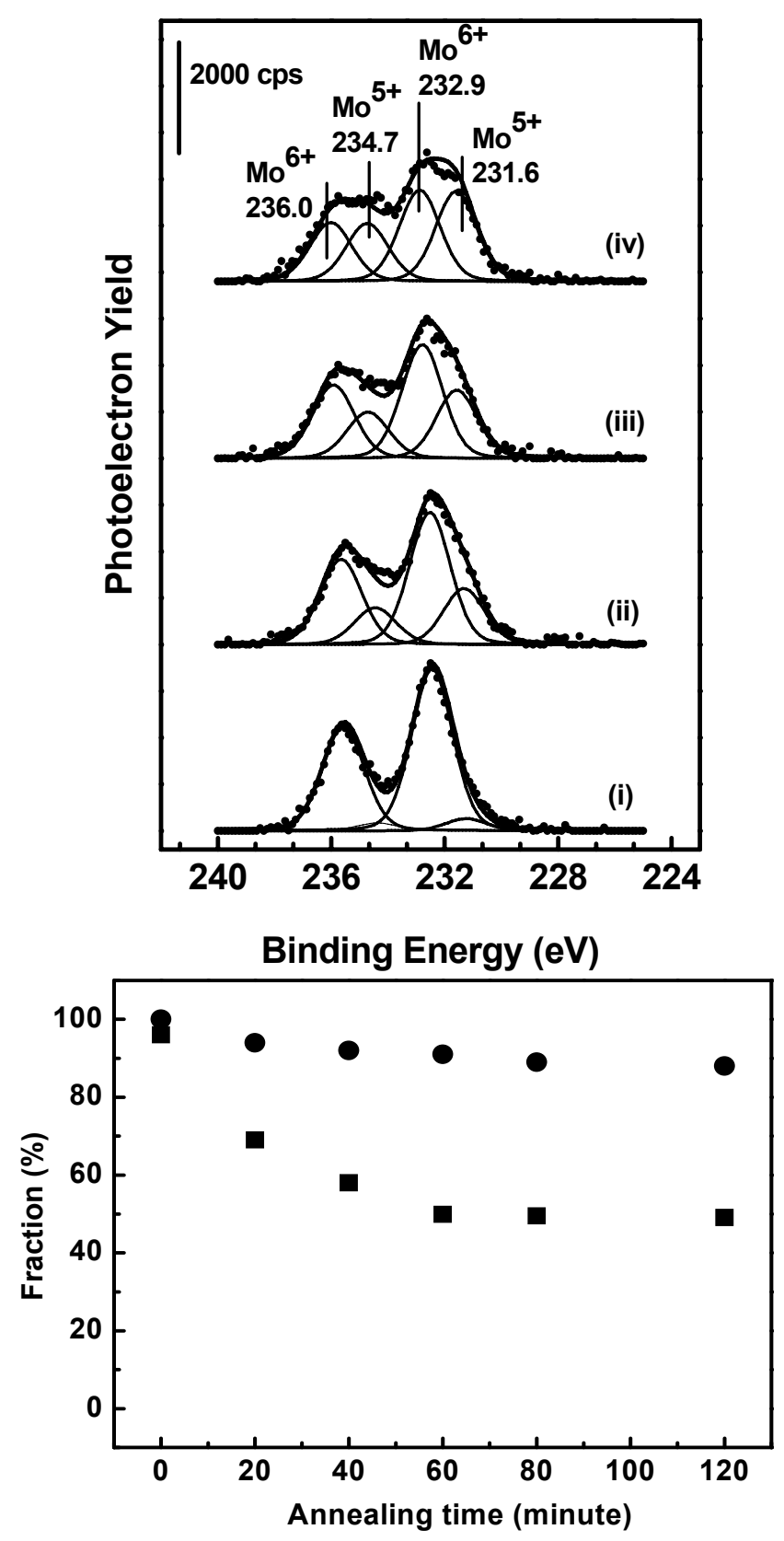

Figure 3. X-ray photoelectron data for the $\mathrm{Mo} 3 \mathrm{~d}$ region showing the reduction of $\mathrm{MoO}_{3}$ nanostructures on $\mathrm{Au}(111)$ prepared by iterative dosing of $\mathrm{Mo}(\mathrm{CO})_{6}$ and $\mathrm{NO}_{2}$. (a) Spectra for: (i) as-prepared material; and followed by annealing to $650 \mathrm{~K}$ for (ii) $20 \mathrm{~min}$; (iii) $40 \mathrm{~min}$; (iv) 60 min. All spectra were obtained at room temperature. (b) Corresponding data showing the fraction of Mo in the $6^{+}$oxidation state (square) and the fraction of Mo remaining on the surface (circle) referenced to the as-prepared sample.

Selective Thermal Reduction of Single-layer $\mathrm{MoO}_{3} \ldots$, Deng, etal. SUSC-D—06-00862 


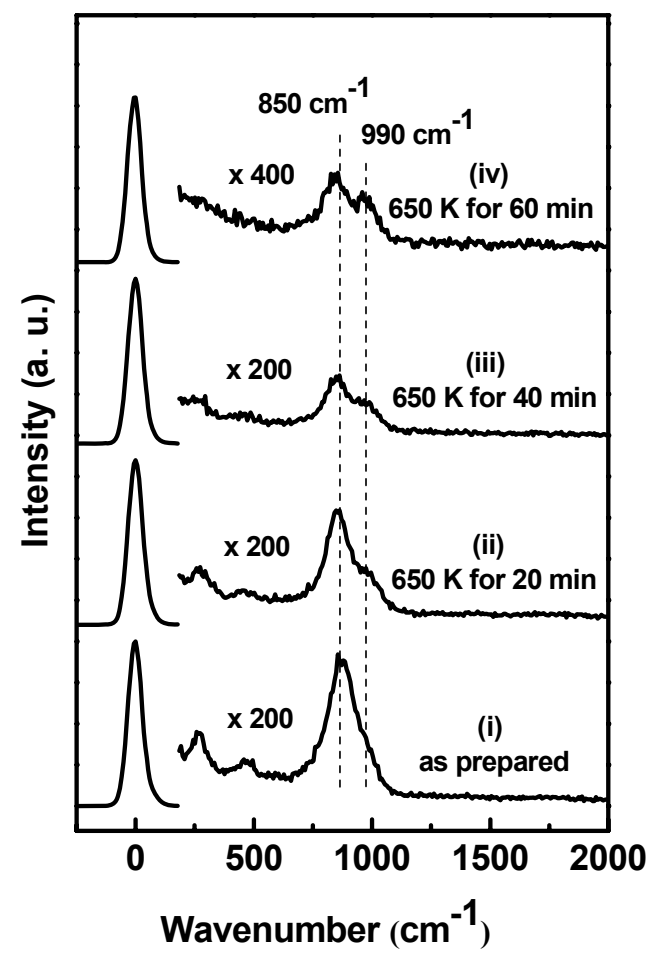

Figure 4. Vibrational (HREEL) spectra obtained after preparing $\mathrm{MoO}_{3}$ nanostructures on $\mathrm{Au}(111)$ by 16 iterations of dosing. The spectrum (i) is taken after the formation of $\mathrm{MoO}_{3}$ without further annealing. The top three spectra are obtained after annealing to $650 \mathrm{~K}$ for (ii) 20 minute, (iii) 40 minute and (iv) 60 minute. The FWHM is typically $\sim 70 \mathrm{~cm}^{-1}$. All spectra were collected at room temperature.

Selective Thermal Reduction of Single-layer $\mathrm{MoO}_{3} \ldots$, Deng, etal. SUSC-D—06-00862 


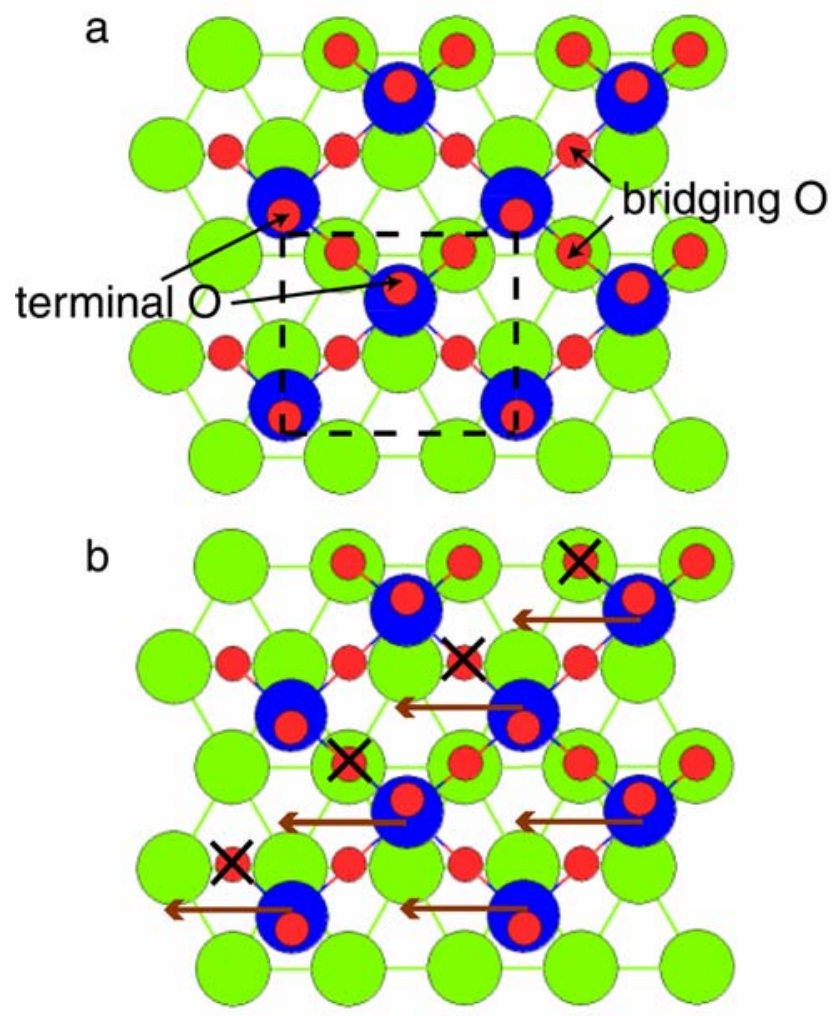

Figure 5. (a) Atomic structure of $\mathrm{MoO}_{3}$ monolayer on $\mathrm{Au}(111)$. (b) Schematic showing the formation of shear defects. The dotted black box in (a) denotes the $c(4 \times 2)$ unit cell. The structure is repeated periodically in the plane of the Au surface. Only the top Au layer is shown (out of a total of 6 layers), as indicated by the hexagonal lattice of green circles. The [1-10] direction of $\mathrm{Au}$ is indicated. Mo and $\mathrm{O}$ are represented respectively by large blue and small red circles. Terminal $\left(\mathrm{O}_{t}\right)$ and bridging $\mathrm{O}^{\prime} \mathrm{s}\left(\mathrm{O}_{\mathrm{a}}\right.$ and $\left.\mathrm{O}_{\mathrm{b}}\right)$ are labeled in (a). The subscripts for $\mathrm{O}_{a}$ and $\mathrm{O}_{\mathrm{b}}$ indicate if the bridging $\mathrm{O}$ is on an atop or bridging site of the $\mathrm{Au}(111)$ surface. The schematic in (b) shows how the oxide structure can 'shear' in the direction of the arrow, to accommodate the loss of a row of bridging O's as marked by black crosses.

Selective Thermal Reduction of Single-layer $\mathrm{MoO}_{3} \ldots$, Deng, etal. SUSC-D—06-00862 


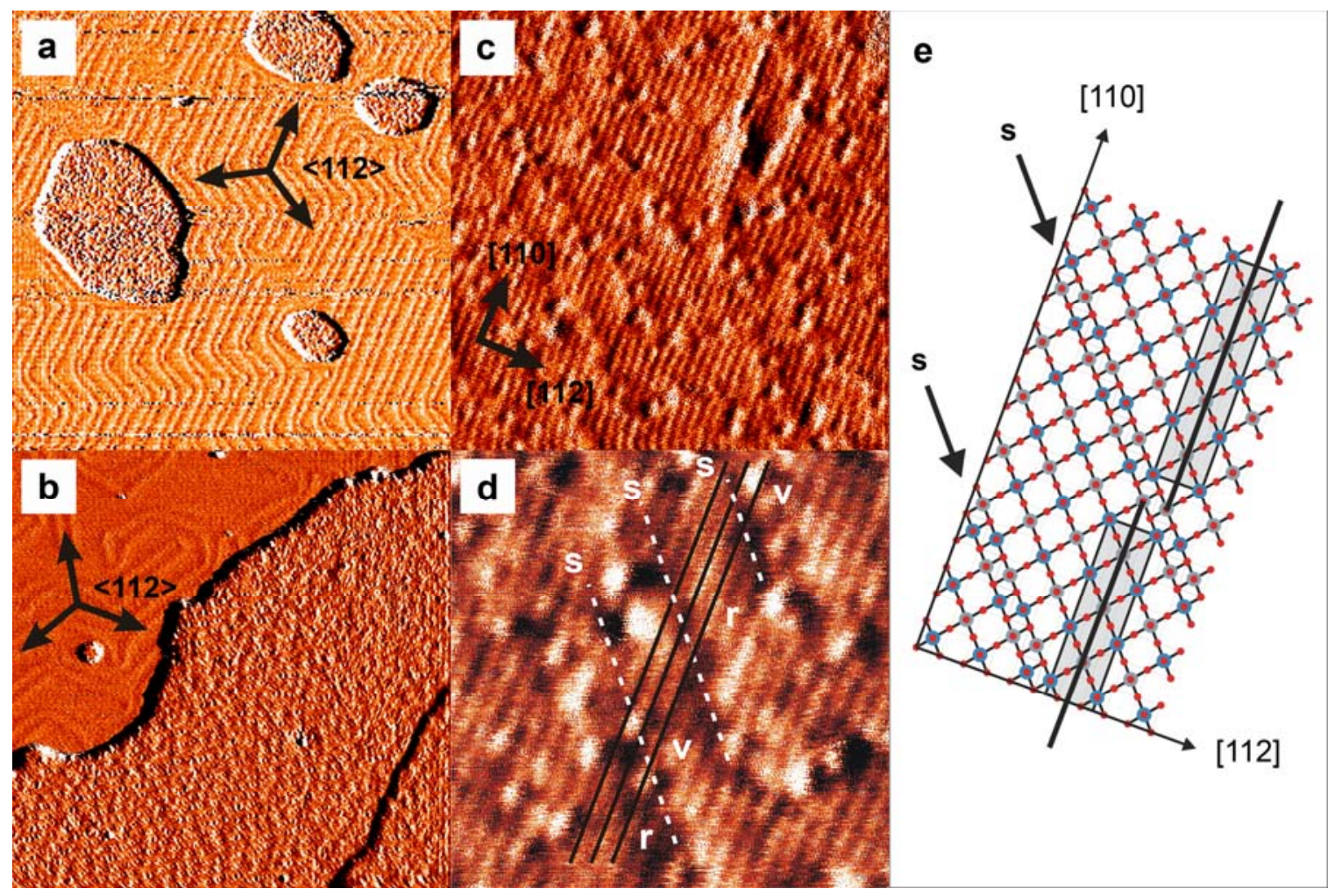

Figure 6. STM images collected from $\mathrm{MoO}_{3}$-covered $\mathrm{Au}(111)$ surfaces before (a) and after annealing at $\mathrm{T}>650 \mathrm{~K}$ (b-d): (a) Stoichiometric $\mathrm{MoO}_{3}$ islands on $\mathrm{Au}(111)$ prepared by PVD of Mo and subsequent oxidation with $\mathrm{NO}_{2}$ at $500 \mathrm{~K}$. The apparent island height is $\sim 0.5 \mathrm{~nm}$ ( $I-$ channel, $125 \mathrm{~nm} \times 125 \mathrm{~nm}$ ). (b) Reduced Mo oxide island formed after annealing at $\mathrm{T}>650 \mathrm{~K}$ (I- channel, $85 \mathrm{~nm}$ x $85 \mathrm{~nm}$ ). Note the increase in size of the Mo oxide island. (c) Higher magnification STM image of the reduced Mo oxide island shown in (b). Despite the appearance of defects, the surface corrugation on top of the island is less than $0.1 \mathrm{~nm}$ demonstrating the 2D character of the structure (Z- channel, $17 \mathrm{~nm} \times 17 \mathrm{~nm}$, vertical color scale is $0-0.125 \mathrm{~nm})$. (d) Extended, one-dimensional defects marked by dashed lines $(s)$ appear on top of the Mo oxide islands and separate regions shifted by half a lattice constant. The full lines serve as a visual guide to demonstrate the lattice shift from a valley $(v)$ to a ridge $(r)$ position on opposite sites of the defect $(s)$ ( $I$ - channel, $8 \mathrm{~nm}$ x $8 \mathrm{~nm}$. (e) Structural model of a reduced single-layer $\mathrm{MoO}_{3}$ island. Note that the presence of shear planes causes a shift of the $\mathrm{MoO}_{3}$ lattice by half a lattice constant between regions separated by a shear defect.

Selective Thermal Reduction of Single-layer $\mathrm{MoO}_{3} \ldots$, Deng, etal. SUSC-D_06-00862 

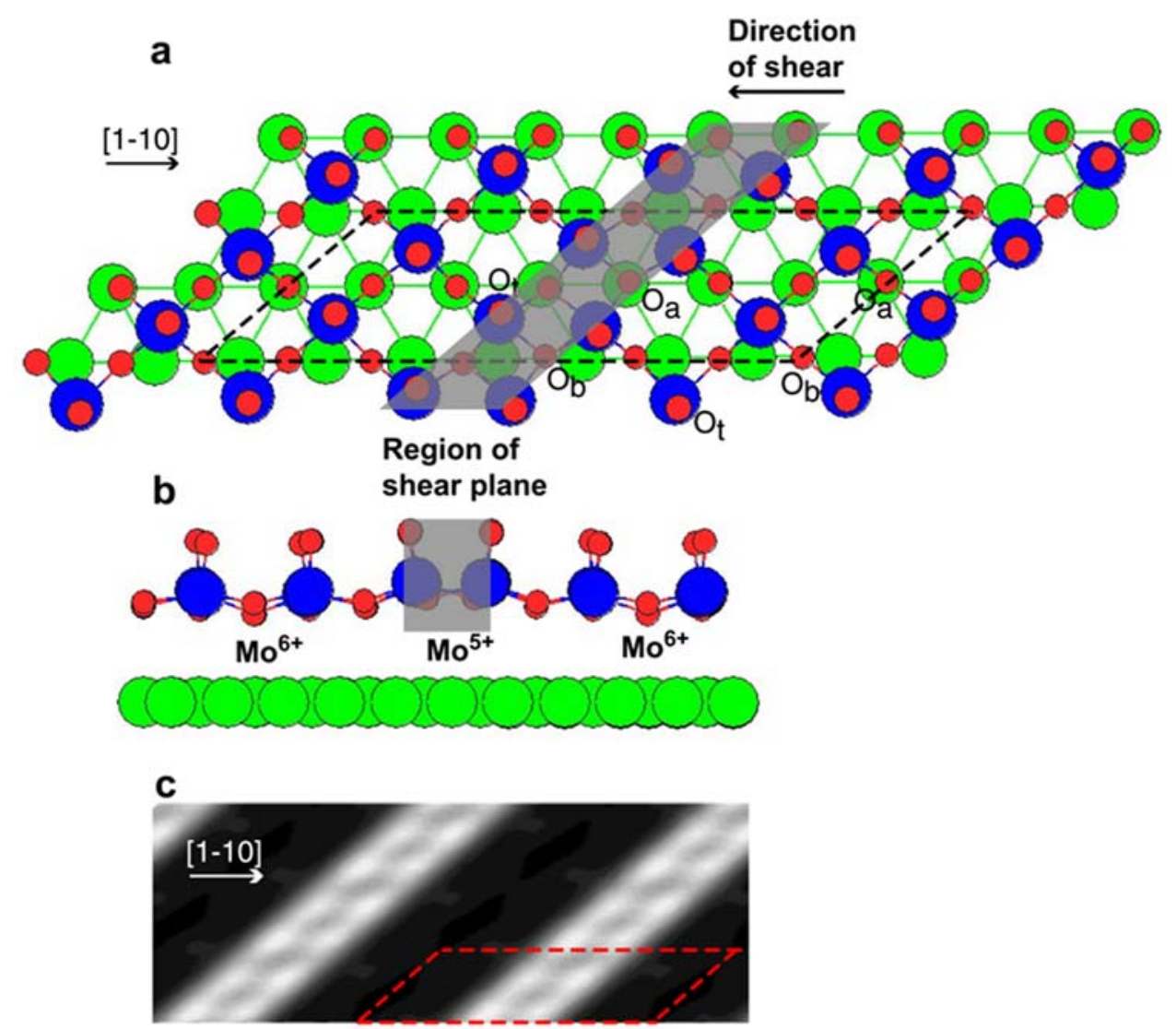

Figure 7. Relaxed shear defect structure, (a) top view and (b) side view. (c) STM simulation with a sample bias of $1.5 \mathrm{~V}$, with Gaussian smearing (standard deviation of $1.2 \AA$ ) to take into account tip convolution effects. The unit cell used in this model is indicated by a dashed black box in (a) and a dashed red box in (c). The defect region is shaded in gray in (a) and (b). The bright area in the STM simulation (c) corresponds to the shear plane defect. Mo atoms in the defect are raised by $\sim 0.3 \AA$ relative to those away from the defect, which are in turn $\sim 3.7 \AA$ above the top Au layer (6\% more than corresponding height in the perfect oxide (Figure 5)).

Selective Thermal Reduction of Single-layer $\mathrm{MoO}_{3} \ldots$, Deng, etal. SUSC-D—06-00862 


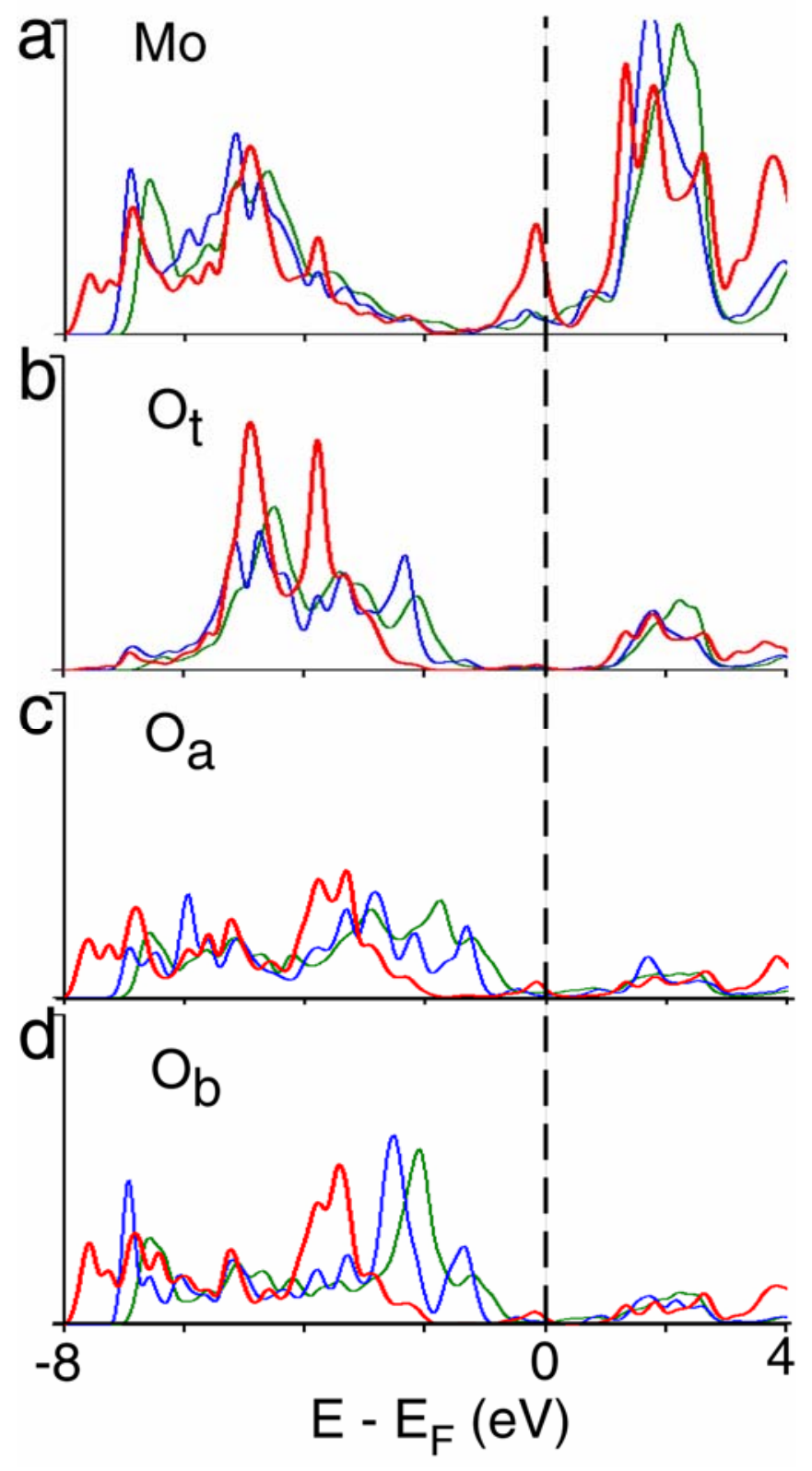

Figure 8. Electronic densities of states projected onto (a) Mo, (b) $\mathrm{O}_{t}$, (c) $\mathrm{O}_{\mathrm{a}}$ and (d) $\mathrm{O}_{\mathrm{b}}$, summed over s, p and d contributions. Red (thickest), blue and green (finest) curves respectively represent atoms at the shear defect, atoms mid-way between the defects in the reduced oxide (Figure 7), and atoms in the fully oxidized oxide (Figure 5).

Selective Thermal Reduction of Single-layer $\mathrm{MoO}_{3} \ldots$, Deng, etal. SUSC-D_06-00862 


\section{References}

1. Quek, S.Y., M.M. Biener, J. Biener, C.M. Friend, and E. Kaxiras, Tuning electronic properties of novel metal oxide nanocrystals using interface interactions: $\mathrm{MoO}_{3}$ monolayers on Au(111). Surface Science Letters, 2005. 577: p. L71-L77.

2. Henrich, V.E. and P.A. Cox, The Surface Science of Metal Oxides. 1994, Cambridge: Cambridge University Press.

3. Spencer, P.J., Process and Catalyst for the Production of Formaldehyde from Methane. United States Patent, 1986. 4,607,127(August 19).

4. Spencer, N.D. and C.J. Pereira, Partial oxidation of $\mathrm{CH}_{4}$ to $\mathrm{HCHO}$ over a $\mathrm{MoO}_{3}-\mathrm{SiO}_{2}$ catalyst: A kinetic study. AIChE Journal, 1987. 33(11): p. 1808-1812.

5. Spencer, N.D., Partial Oxidation of Methane to Formaldehyde by Means of Molecular Oxygen. Journal of Catalysis, 1988. 109: p. 187-197.

6. Banares, M.A., N.D. Spencer, M.D. Jones, and I.E. Wachs, Effect of Alkali Metal Cations on the Structure of $\mathrm{Mo}(\mathrm{VI}) / \mathrm{SiO}_{2}$ Catalysts and Its Relevance to the Selective Oxidation of Methane and Methanol. J.Catal., 1994. 146: p. 204-210.

7. SalehAlhamed, Y.A., R.R. Hudgins, and P.L. Silveston, Role of water vapor in the partial oxidation of propene. Journal of Catalysis, 1996. 161(1): p. 430-440.

8. Deiner, L.J., A.S.Y. Chan, M.A. Sheehy, and C.M. Friend, The Reaction of $\mathrm{CH}_{3} \mathrm{NO}_{2}$ on O-covered Mo(110): The Effect of Oxygen on Product Distribution. Surface Science Letters, 2004. 555(1-3): p. L127-L132.

9. Surnev, S., G. Kresse, M.G. Ramsey, and F.P. Netzer, Novel interface-mediated metastable oxide phases: Vanadium oxides on Pd(111). Physical Review Letters, 2001. 8708(8).

10. Costello, C.K., J. Guzman, J.H. Yang, Y.M. Wang, M.C. Kung, B.C. Gates, and H.H. Kung, Activation of Au/gamma-Al2O3 catalysts for CO oxidation: Characterization by $X$-ray absorption near edge structure and temperature programmed reduction. Journal of Physical Chemistry B, 2004. 108(33): p. 12529-12536.

11. Liu, Z.P., C.M. Wang, and K.N. Fan, Single gold atoms in heterogeneous catalysis: Selective 1,3-butadiene hydrogenation over Au/ZrO2. Angewandte Chemie-International Edition, 2006. 45(41): p. 6865-6868.

12. Wang, X.a.W., IE, Designing the Activity/Selectivity of Surface Acidic, Basic and Redox Active Sites in the Supported K2O-V205/A12O3 Catalytic System. Catalysis Today, 2004. 96(4): p. 211-222.

13. Biener, M.M., Friend C.M., Heteroepitaxial Growth of Novel $\mathrm{MoO}_{3}$ Nanostructures on $A u(111)$. Surface Science Letters, 2004. 559(2-3): p. L173-L179.

14. Biener, M.M., J. Biener, R. Schalek, and C.M. Friend, Growth of nanocrystalline MoO3 on Au(111) studied by in-situ STM. J. Chem. Phys., 2004. 121(23): p. 12010-12016.

15. Chang, Z.P., Z. Song, G. Liu, J.A. Rodriguez, and J. Hrbek, Synthesis, electronic and chemical properties of MoOx clusters on Au(111). Surface Science, 2002. 512(1-2): p. L353-L360.

16. Song, Z., T. Cai, Z. Chang, G. Liu, J.A. Rodriguez, and J. Hrbek, Molecular Level Study of the Formation and the Spread of MoO3 on Au (111) by STM and XPS. J. Am. Chem. Soc., 2003. 125: p. 8059-8066.

Selective Thermal Reduction of Single-layer $\mathrm{MoO}_{3} \ldots$, Deng, etal. SUSC-D—06-00862 
17. Kresse, G. and J. Furthmuller, Efficient iterative schemes for ab initio total-energy calculations using a plane-wave basis set. Phys. Rev. B, 1996. 54(16): p. 11169-11186.

18. Bursill, L.A., Crystallographic shear in molybdenum trioxide. Proc. Roy. Soc. A, 1969. 311: p. 267-290.

Selective Thermal Reduction of Single-layer $\mathrm{MoO}_{3} \ldots$, Deng, etal. SUSC-D—06-00862 